\title{
No balance between glutamate+glutamine and GABA+ in visual and motor cortices of the human brain
}

\author{
ABBREVIATED TITLE: NO BALANCE BETWEEN GLX AND GABA IN VISUAL AND \\ MOTOR CORTICES
}

Reuben Rideaux

Department of Psychology, Downing Street, University of Cambridge, UK reuben.rideaux@gmail.com

Conflict of interest statement: The authors declare no competing interests.

Acknowledgements: The work was supported by the Leverhulme Trust (ECF-2017573), the Issac Newton Trust (17.08(0)). 


\section{ABSTRACT}

Theoretical work, supported by electrophysiological evidence, asserts that a

3 balance between excitation and inhibition (E/I) is critical for healthy brain function. In

4 magnetic resonance spectroscopy (MRS) studies, the ratio of excitatory (glutamate)

5 and inhibitory ( $Y$-aminobutyric acid, GABA) neurotransmitters is often used as a proxy

6 for this E/I balance. Recent MRS work found a positive correlation between GABA+

7 and Glx (glutamate+glutamine) in medial parietal cortex, providing validation for this

8 proxy and supporting the link between the E/I balance observed in electrophysiology

9 and that detected with MRS. Here we assess the same relationship, between GABA+

10 and Glx, in primary visual and motor cortices of resting male and female human

11 participants. We find moderate to strong evidence that there is no positive correlation

12 between these neurotransmitters in either location. We show this holds true when

13 controlling for a range of other factors (i.e., demographics, signal quality, tissue

14 composition, other neurochemicals) and regardless of the state of neural activity (i.e.,

15 resting/active). These results show that there is no brain-wide balance between

16 excitatory and inhibitory neurotransmitters and indicates a dissociation between the

$17 \mathrm{E} / \mathrm{l}$ balance observed in electrophysiological work and the ratio of MRS-detected neurotransmitters. 


\section{INTRODUCTION}

20

Balanced excitatory and inhibitory activity (i.e., E/l balance) is a canonical feature in models of healthy brain (Marín, 2012; Shadlen \& Newsome, 1994; Van Vreeswijk \& Sompolinsky, 1996; Vogels \& Abbott, 2009), which is supported by a large body of empirical evidence, primarily from electrophysiological experiments. Diverse external stimulation, such as a bar of light (Anderson, Carandini, \& Ferster, 2000), a whisker deflection (Wilent \& Contreras, 2005), a brief tone (Wehr \& Zador, 2003), or an odour (Poo \& Isaacson, 2009), all evoke concurrent excitatory and inhibitory activity, which is thought to bestow neurons with sharper tuning properties (Higley \& Contreras, 2006; Wilent \& Contreras, 2005). The phenomenon can even be observed during periods of spontaneous activity and is likely responsible for maintaining equilibrium throughout the brain (Okun \& Lampl, 2008). By contrast, E/I imbalance is implicated in central pathologies including epilepsy (Bradford, 1995; Olsen \& Avoli, 1997), autism spectrum disorder (Chao et al., 2010; Markram \& Markram, 2010; Rubenstein \& Merzenich, 2003; Vattikuti \& Chow, 2010), and schizophrenia (Kehrer, Maziashvili, Dugladze, \& Gloveli, 2008).

Magnetic resonance spectroscopy (MRS) can be used to measure in vivo concentrations of primary excitatory (glutamate) and inhibitory ( $\gamma$-aminobutyric acid, GABA) neurotransmitters within the brain. It is common practice in MRS studies to report the ratio of these neurotransmitters as an index of E/l balance. This proxy has been used to study a range of healthy brain functions including decision-making (Bezalel, Paz, \& Tal, 2019), visual learning (Shibata et al., 2017), volitional control (Koizumi, Lau, Shimada, \& Kondo, 2018), memory (Bang et al., 2018; Takei et al., 2016), and visual contrast sensitivity (Ip, Emir, Parker, \& Bridge, 2018). It has also been used to investigate other neuroimaging signals, such as the default mode network activity (Gu, Hu, Chen, He, \& Yang, 2019; Kapogiannis, Reiter, Willette, \& Mattson, 2013). In the context of theories relating neuropsychiatric conditions to $E / I$ imbalance, GABA and glutamate or Glx (a complex comprising glutamate and glutamine) concentrations have been identified as a potential biomarker for autism spectrum disorder and schizophrenia (Brown, Singel, Hepburn, \& Rojas, 2013; Egerton et al., 2012; Horder et al., 2013; Smesny et al., 2015). 
A recent MRS study tested the validity of using the ratio of excitatory and inhibitory neurotransmitters as a proxy for E/I balance (Steel, Mikkelsen, Edden, \&

52 Robertson, 2020). The researchers found that the concentration of Glx and GABA+

53 (GABA + co-edited macromolecules) in medial parietal cortex was positively correlated

54 across a large cohort of healthy participants. This evidence suggests an association

55 between electrophysiological and MR spectroscopic observations of E/l balance.

56 However, if these measurements reflected a common neural phenomenon (i.e., E/I

57 balance), we would expect to observe a positive correlation between GABA+ and GIx

58 at other cortical regions where concomitant excitatory and inhibitory activity has been

59 reported. Thus, to assess the regional specificity of this association, we tested for a

60 relationship between GABA+ and GIx in two different cortical locations: early visual and motor cortices. These brain regions are an ideal testbed of this association, as numerous electrophysiological studies have demonstrated their E/I balance (Anderson et al., 2000; Dehghani et al., 2016; Maffei \& Turrigiano, 2008; Priebe \& Ferster, 2005; Wilent \& Contreras, 2005; Xue, Atallah, \& Scanziani, 2014).

In contrast to Steel et al. (2020), we find no evidence for a relationship between GABA+ and Glx across participants in either visual or motor cortices. Post-hoc

67 Bayesian analyses reveal moderate to strong evidence in favour of the null hypothesis. We further show that the same (null) result is found in visual cortex even during visual stimulation, indicating that the discrepancy between the results from medial parietal cortex and visual/motor cortices cannot be explained by differences in overall neural activity. These findings show that there is no canonical brain-wide balance between be the exception, rather than the rule. Moreover, the results indicate a dissociation between the $E / l$ balance observed using other techniques (e.g., electrophysiology) and the ratio of excitatory and inhibitory neurotransmitters detected with MRS. 
Data collection. Legacy data from previous studies were combined. In these studies, male and female human participants underwent MR spectroscopic acquisition targeting visual ( $n=58$; 31 women; mean age=24.4; age range=19-40; Rideaux, 2020; Rideaux, Goncalves, \& Welchman, 2019; Rideaux \& Welchman, 2018), motor ( $n=50$; 24 women; mean age=24.3; age range=19-36; Rideaux \& Welchman, 2018) cortices while at rest. During the acquisition, the lights in the room were turned off and participants were instructed to close their eyes. In addition to this, data from visual cortex of participants $(n=31 ; 17$ women; mean age=24.9; age range=19-40; Rideaux et al., 2019) who received visual stimulation during acquisition was analysed as a control. All data were collected on the same vendor at the same site. All participants were screened for contra-indications to MRI prior to data collection. All experiments were conducted in accordance with the ethical guidelines of the Declaration of Helsinki and were approved by the University of Cambridge ethics committee and all participants provided informed consent.

Data acquisition. Magnetic resonance scanning was conducted on a 3T Siemens Prisma equipped with a 32-channel head coil. Anatomical T1-weighted images were acquired for voxel placement with an MP-RAGE sequence. For detection of GABA+, spectra were acquired using a MEGA-PRESS sequence (Mescher, Merkle, Kirsch, Garwood, \& Gruetter, 1998): TE=68 ms, TR=3000 ms; 256 or 400 transients of 2048 data points were acquired, 16 water-unsuppressed transients were additionally acquired; a 14.28 ms Gaussian editing pulse was applied at 1.9 (ON) and 7.5 (OFF) ppm. Water suppression was achieved using variable power with optimized relaxation delays (VAPOR; Tkáč and Gruetter, 2005) and outer volume suppression. Automated shimming with 3D GRE, followed by manual shimming, was conducted to achieve approximately $12 \mathrm{~Hz}$ water linewidth. All MRS data were analysed from their DICOM 102 format.

Spectra were acquired from locations targeting visual and motor cortices (Fig. 1a). The voxel targeting visual cortex $(n=40,3 \times 3 \times 2 \mathrm{~cm} ; n=29,2.5 \times 2.5 \times 2.5 \mathrm{~cm})$ was positioned medially in the occipital lobe with the lower face aligned with the cerebellar tentorium. The voxel targeting motor cortex $(3 \times 3 \times 2 \mathrm{~cm})$ was centred on the 'hand 
107

108

109

110

111

112

113

114

115

116

117

118

119

120

121

122

123

knob' area of the precentral gyrus and aligned to the upper surface of the brain in the sagittal and coronal planes. The coordinates of the voxel location were used to draw a mask on the anatomical T1-weighted image to calculate the fractional volume of grey matter, white matter, and cerebrospinal fluid within each voxel. Tissue segmentation was performed using the Statistical Parametric Mapping toolbox for MATLAB (SPM12, www.fil.ion.ucl.ac.uk/spm; Ashburner \& Friston, 2005).

The conditions of the 'visual stimulation' control data acquisition were the same as those described in the resting visual cortex dataset, with the exception that participants viewed black and white random-dot kinetograms during the acquisition. In particular, participants performed an attentionally demanding vernier task at fixation while an annulus comprising black and white dots, with a fan-shaped threedimensional profile, was presented around fixation. Participants were required to monitor fixation for the lines used to perform the vernier task, which appeared at pseudorandom intervals throughout the scan. The random-dot kinetograms were presented for $1.8 \mathrm{~s}$ and separated by $0.2 \mathrm{~s}$ inter-stimulus-intervals throughout the scan. For a detailed description of the stimulus and presentation procedure, see the 'mixed-polarity' condition in Rideaux et al. (2019).

a

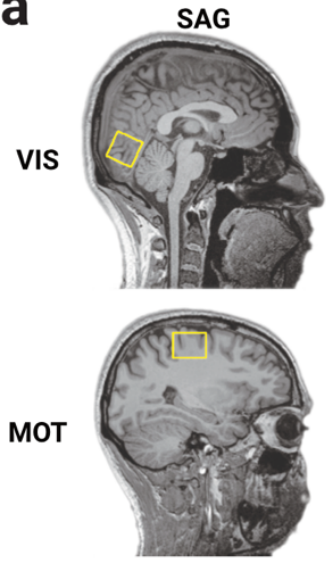

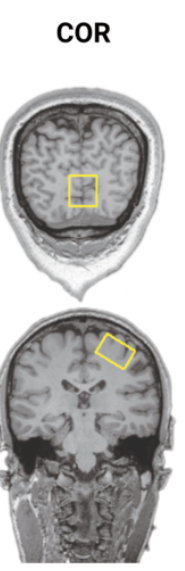

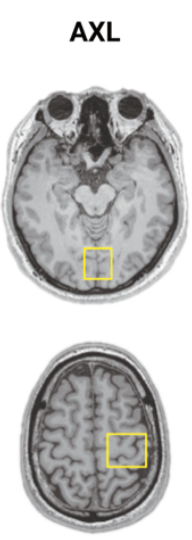

b

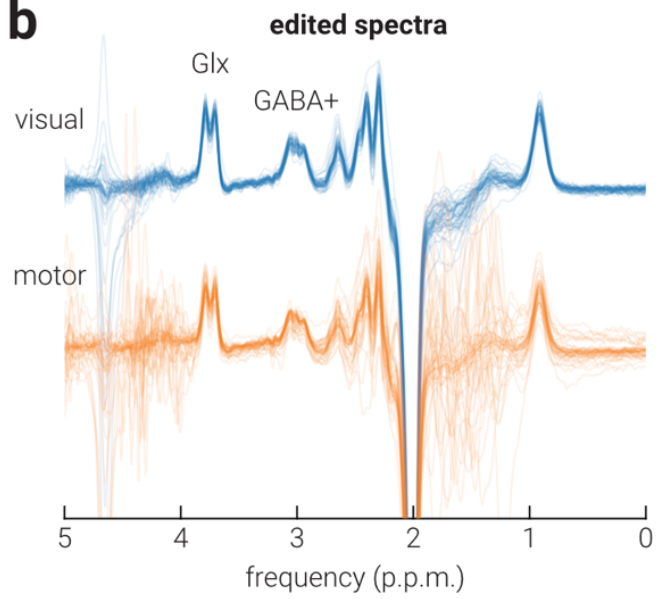

Figure 1. Voxel locations and average edited spectra. a) Sagittal (SAG), coronal (COR), and axial (AXL) views of representative MRS voxel placement for visual (VIS) and motor (MOT) cortices on a T1-weighted structural image. b) Average edited spectra for resting visual and motor cortices.

124 Data processing. Spectral pre-processing and quantification was conducted in 125 MATLAB using a combination of Gannet v3.1 (Edden et al., 2014) and in-house scripts. Prior to alignment, subspectra were zero-filled to a spectral resolution of 0.061

$127 \mathrm{~Hz} /$ point and 3-Hz exponential line-broadening was applied. Frequency and phase parameter estimates were obtained by modelling the total creatine (tCr; creatine and 
phosphocreatine) signal, then these parameters were used to align subspectra to a common frequency and phase. Note that we found that same pattern of results when Spectral Registration was used to align spectra. Estimates of signal area and full-width

132 at half-maximum (FWHM) were also obtained, and subspectra (and their corresponding ON/OFF subspectra) with parameter estimates $>3$ standard deviations (s.d.; the default cut-off applied in Gannet) from the mean within a scan were omitted from further analysis.

Total creatine and total $\mathrm{N}$-acetyl aspartate (tNAA; N-acetyl aspartate and Nacetyl aspartyl glutamate) signal intensity were determined by fitting a Lorentzian model to the average OFF spectra at $3.0 \mathrm{ppm}$ and $2.0 \mathrm{ppm}$, respectively. The average ON and OFF spectra were subtracted to produce the edited spectrum (Fig. 1b), from which GABA+ (3 ppm) and Glx (3.8 ppm) signal intensity were modelled. Glx and GABA+ were fit using a double-Gaussian model. Water signal intensity was determined by fitting a Lorentzian model to the average water-unsuppressed spectra at $4.9 \mathrm{ppm}$. All neurochemical signal intensities were calculated as the area of the fitted peak(s) and expressed in institutional units (i.u.) using the unsuppressed water signal as an internal concentration reference. The assumed relaxation and density parameters of water and GABA are described in (Mikkelsen et al., 2019). The assumed longitudinal relaxation times of Glx, tCr, tNAA, and Cho were $1.23 \mathrm{~s}, 1.35 \mathrm{~s}$, $1.41 \mathrm{~s}$, and $1.19 \mathrm{~s}$, respectively (Posse et al., 2007). The assumed transverse relaxation times of these metabolites were $0.18 \mathrm{~s}, 0.15 \mathrm{~s}, 0.25 \mathrm{~s}, 0.21 \mathrm{~s}$, respectively (Ganji et al., 2012).

Statistical analyses. Statistical analyses were conducted in MATLAB (The MathWorks, Inc., Matick, MA). Prior to correlational analyses, we screened the data by omitting ( $n=3$, all from motor cortex group) participants with GABA+ or Glx values $>2.5$ s.d. from the mean, in line with (Steel et al., 2020). We sought to determine whether the concentration of GABA+ and Glx were related across different brain regions. To test this, we computed the Pearson correlation between GABA+ and GIx for all spectra. Next, to test whether the relationship GABA+ and Glx could be explained by confounding factors we systematically regressed out the influence of factors that could potentially account for the observed relationship using a linear mixed effects model. We then computed the Pearson correlation between the residuals after 
165 controlling for the confounding factors. We reasoned that if the residual values 166 remained correlated, the relationship between GABA+ and Glx held when controlling 167 for these confounding variables. To establish the influence of confounding factors, we 168 considered each factor separately and calculated the significance of the change in 169 correlation by comparing z-scored correlation coefficients. Power analyses were 170 conducted in GPower (www.gpower.hhu.de; Erdfelder, Faul, \& Buchner, 1996). 171 Bayesian analyses were conducted in JASP (www.jasp-stats.org; Wagenmakers et 172 al., 2018). 


\section{RESULTS}

174 No balance between GABA+ and GIx. We found no evidence for a relationship 175 between GABA+ and Glx across participants in either visual ( $\left.r_{58}=-.09, P=.493\right)$ or 176 motor cortices ( $\left.r_{47}=-.05, P=.762\right)$. Power analyses, based on the correlation between 177 GABA+ and Glx in medial parietal cortex found by Steel et al. (2020) $(r=.40)$, showed 178 that the sample sizes tested here had a $94 \%$ and $89 \%$ probability of detecting the 179 same relationship if it existed in visual and motor cortices, respectively. There are 180 multiple factors that may influence the quantification of GABA+ and/or Glx with MRS. 181 It is possible that a correlation between these neurotransmitters in visual and/or motor 182 cortices was masked by an additional moderating factor. To test this possibility, we 183 systematically assessed the relationship between GABA+ and Glx after regressing out 184 a range of possible moderating factors. The results of these analyses are shown in 185 Table 1. 
bioRxiv preprint doi: https://doi.org/10.1101/2021.03.02.433628; this version posted March 2,2021 . The copyright holder for this preprint (which was not certified by peer review) is the author/funder, who has granted bioRxiv a license to display the preprint in perpetuity. It is made available under aCC-BY 4.0 International license.

a
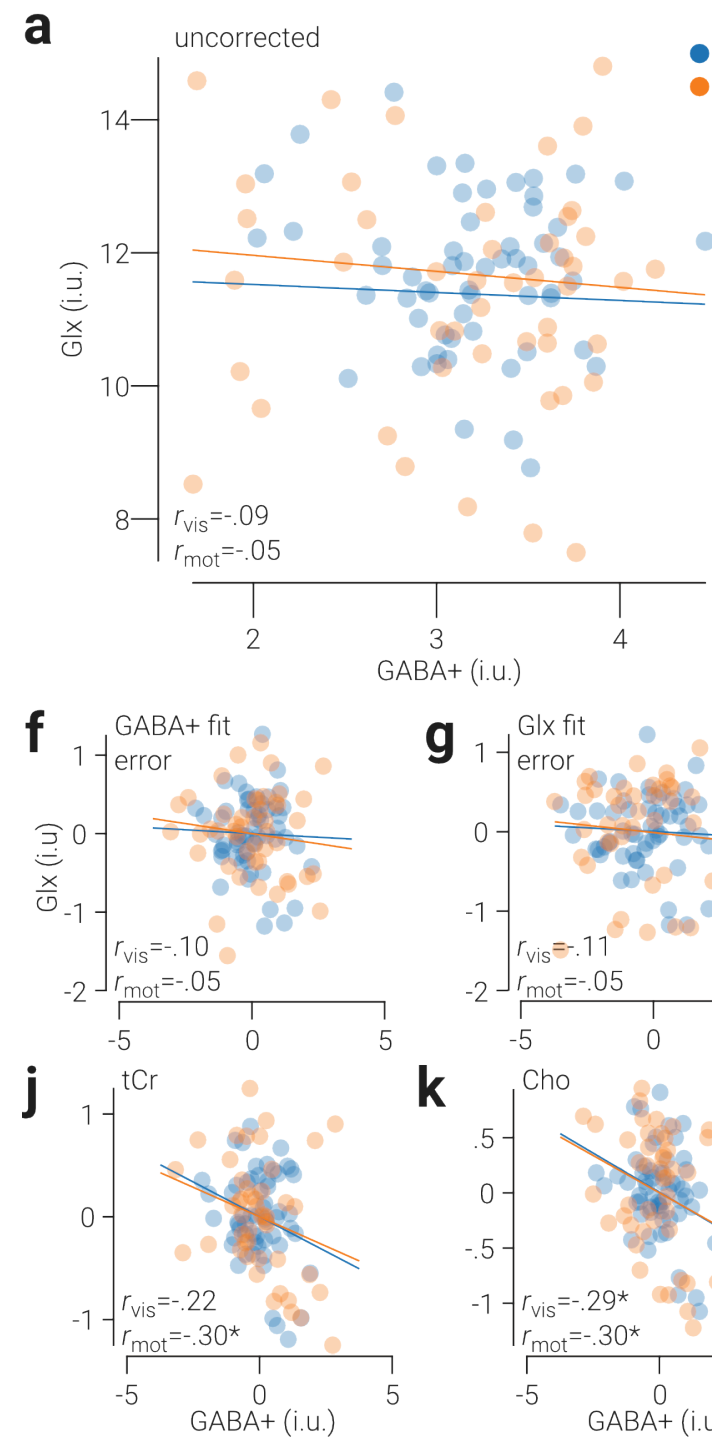

k
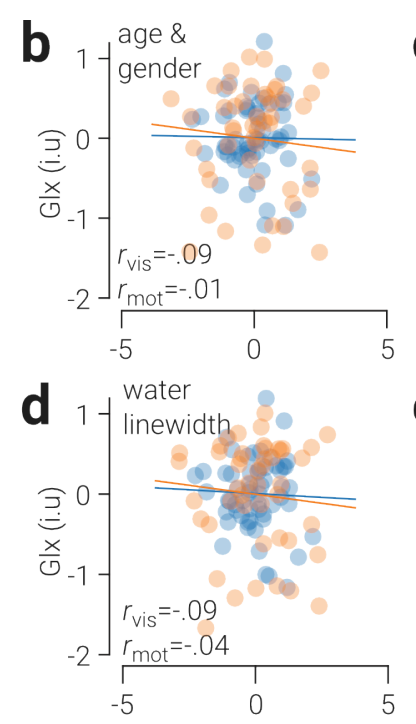

h
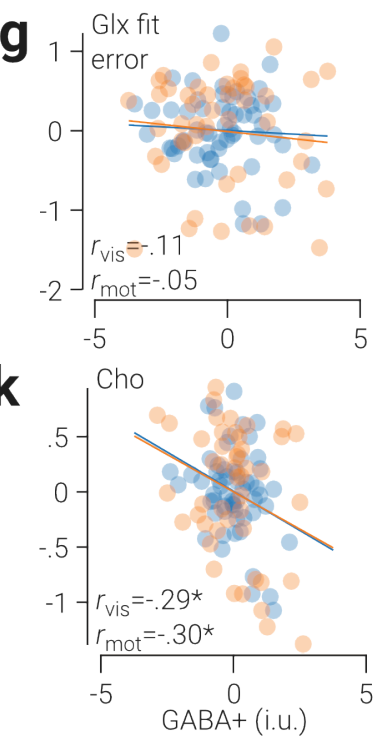

C

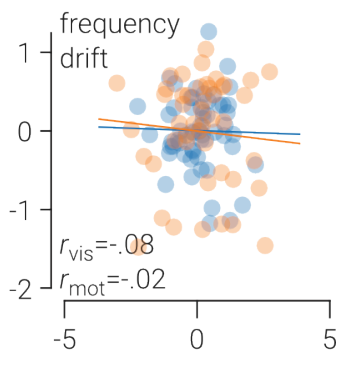

e
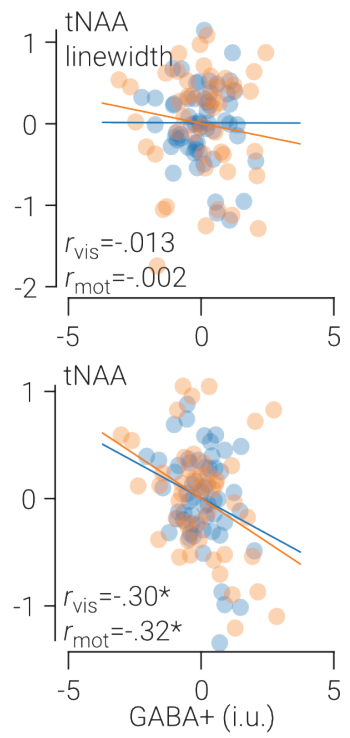

Figure 2. No balance between GABA+ and GIx in visual and motor cortices. a) Glx concentration as a function of GABA+ concentration in early visual and motor cortices. $\mathbf{b}-\mathbf{g}$ ) The same as (a), after controlling for $\mathbf{b}$ ) age and gender, c) frequency drift, d) water linewidth, e) tNAA linewidth, f) GABA+ fit error, g) Glx fit error, h) grey matter, i) tNAA concentration, j) tCr concentration, $\mathbf{k}$ ) Cho concentration, and I) water concentration. Glx and GABA+ values are referenced to water and expressed in institutional units (i.u.). Lines indicate best linear fit, and cyan and orange colours indicate data from visual and motor cortices, respectively. Asterisks indicate correlation coefficients where $p<05$. 
Table 1. List of confounding factors tested.

\begin{tabular}{lcc}
\hline \multirow{2}{*}{ Confounding factor } & \multicolumn{2}{c}{ Correlation of GABA+ and Glx residuals } \\
\cline { 2 - 3 } age and gender & $r=-.09, p=.494$ & motor \\
frequency drift & $r=-.08, p=.563$ & $r=-.02, p=.879$ \\
water linewidth & $r=-.09, p=.504$ & $r=-.04, p=.804$ \\
tNAA linewidth & $r=-.13, p=.334$ & $r=-.002, p=.991$ \\
GABA+ fit error & $r=-.10, p=.466$ & $r=-.05, p=.763$ \\
Glx fit error & $r=-.11, p=.417$ & $r=-.05, p=.727$ \\
grey matter & $r=-.10, p=.467$ & $r=-.03, p=.830$ \\
tNAA concentration & $r=-.30, p=.021$ & $r=-.32, p=.029$ \\
tCr concentration & $r=-.22, p=.096$ & $r=-.30, p=.040$ \\
Cho concentration & $r=-.29, p=.026$ & $r=-.30, p=.040$ \\
water concentration & $r=-.11, p=.442$ & $r=-.16, p=.269$ \\
\hline
\end{tabular}

188

189

190

191

192

193

194

195

196

197

198

199

200

201

202

203

204

Age and gender. Previous MRS work indicates that demographic characteristics such as age and gender are related to the concentration of GABA+ and Glx. That is, in adults, the concentration of GABA+ and Glx tends to be higher in males (O'Gorman, Michels, Edden, Murdoch, \& Martin, 2011), and decreases with age (Cassady et al., 2019; Gao et al., 2013; Sailasuta, Ernst, \& Chang, 2008). However, we found no significant difference in the relationship between GABA+ and GIx in either visual or motor cortices as a result of controlling for age and gender (Fig. 2 b; visual: $z_{57}=0.001$, $p=.999$; motor: $\left.z_{47}=0.15, p=.884\right)$.

Spectral quality. As both GABA+ and Glx signals were quantified from the same spectra, differences in signal quality may have obscured their relationship. That is, low signal quality could result in systematic misestimation of both GABA+ and Glx, producing a spurious correlation that masks a putative non-spurious relationship between these neurochemicals.

To assess this possibility, we regressed out the differences in three measures of signal quality (frequency drift, tNAA linewidth, and water linewidth) before re-testing the relationship between GABA+ and Glx. Frequency drift can occur from participant head motion or field gradient heating/cooling and can impact quantification of GABA+ 
and Glx by altering the efficiency with which their signals are edited (Harris et al., 2014). The editing efficiency of GABA+ decreases (modestly) with frequency drift in either direction, whereas that of Glx can increase or decrease depending on the drift direction (Harris et al., 2014). Thus, frequency drift could produce either a positive or negative spurious correlation between GABA+ and Glx. However, we found no significant difference in the relationship between GABA+ and Glx in either visual or motor cortices as a result of controlling for frequency drift (Fig. 2c; visual: $z_{57}=0.08$, $p=.940$; motor: $\left.z_{47}=0.11, p=.916\right)$. The linewidth of metabolite peaks provides an indication of signal quality, as larger linewidths are associated with poorer spectral acquisition, e.g., due to participant head motion or inefficient shimming (Harris et al., 2014). However, we found no significant change in the correlation between GABA+ and Glx as a result of controlling for either water (Fig. 2d; visual: $z 57=0.01, p=.990$; motor: $z_{47}=0.04, p=.969$ ) or tNAA linewidth (Fig. 2e; visual: $z_{57}=0.20, p=.845$; motor: $\left.Z_{47}=0.20, p=.838\right)$.

Another measure that can provide an indirect measure of spectral quality is the residual error associated with fitting models to metabolite signals, i.e., fit error. If the fit error is high, this indicates that the model is poorly fit to the signal and can indicate poor signal quality. Thus, we also included the fit error associated with GABA+ and Glx in our regression analysis. However, we found no significant change in the correlation between GABA+ and Glx as a result of controlling for either GABA+ (Fig. 2f; visual: $z_{57}=0.03, p=.975$; motor: $z_{47}=0.00, p=1.0$ ) or Glx fit error (Fig. 2g; visual: $Z_{57}=0.09, p=.929$; motor: $\left.Z_{47}=0.03, p=.974\right)$.

It is challenging to rule out the possibility that signal quality contributed to the lack of a correlation between GABA+ and Glx. In particular, if GABA+ and Glx measurements were inaccurate due to poor signal quality, we would not expect them be correlated. However, if this were true, we would also expect them to be uncorrelated with other metabolite concentrations. To test this possibility, we computed the correlation between GABA+ and Glx and tCr and tNAA. We found that in visual and motor cortices both GABA+ and Glx were significantly positively correlated with $\mathrm{tCr}$ and tNAA (all $p<.05$ ), confirming that the measurements of GABA+ and Glx were sufficiently accurate to detect relationships between metabolites. 
236 Tissue composition. GABA is approximately twice as highly concentrated in grey 237 matter compared to white matter (Petroff, Ogino, \& Alger, 1988), and negligible in 238 cerebrospinal fluid. Thus, inter-individual differences in the fractional tissue 239 composition within the MRS voxel may have obscured a relationship between GABA+ 240 and Glx. However, we found no significant change in the correlation between these 241 neurotransmitters in visual or motor cortices as a result of controlling for the proportion 242 of grey matter (Fig. 2 h; visual: $z_{57}=0.03, p=.976$; motor: $z_{46}=0.06, p=.953$ ). Note that 243 differences in voxel tissue composition may explain the difference in average GABA+ and Glx concentrations observed here and in Steel et al. (2020).

We next tested the possibility that differences in voxel tissue composition obscured a relationship between GABA+ and Glx by applying an alpha-tissue correction method (Harris, Puts, \& Edden, 2015). However, we found that the correlation between these neurotransmitters in visual or motor cortices (visual: $r=-.12$, $p=.364$; motor: $r=-.08, p=.573$ ) did not change significantly as a result of applying this correction (visual: $z_{57}=0.16, p=.876$; motor: $z_{46}=0.18, p=.854$ ). Finally, we applied a tissue-correction method that accounts for differences in relaxation times across the tissue types within a voxel (Gasparovic et al., 2006). In line with the previous results, we found that the correlation between GABA+ and Glx in visual or motor cortices (visual: $r=-.11, p=.425$; motor: $r=-.15, p=.322$ ) did not change significantly as a result of applying this correction (visual: $z_{57}=0.08, p=.937$; motor: $Z_{46}=0.49, p=.628$ ).

Other neurochemicals. Previous work reported that the relationship between GABA+ and Glx in medial parietal cortex is moderated by the concentration of tCr and tNAA (Steel et al., 2020). While we found no significant change in the relationship between GABA+ and Glx as a result of controlling for $\mathrm{tCr}$ (Fig. 2i; visual: $z_{57}=1.16, p=.247$; motor: $Z_{47}=1.33, p=.182$ ) or tNAA (Fig. 2j; visual: $Z_{57}=0.69, p=.487$; motor: $Z_{47}=1.24$, $p=.214$ ), we found a significant negative correlation between GABA+ and Glx in visual cortex, after controlling for the concentration of tNAA ( $\left.r_{58}=-.30, p=.021\right)$, and in motor cortex, after controlling for the concentration of $\operatorname{tCr}\left(r_{47}=-.30, p=.040\right)$ and tNAA $\left(r_{47}=-\right.$ .32, $p=.027)$.

Steel et al. (2020) found no difference in the relationship between GABA+ and Glx in medial parietal cortex as a result of controlling for the concentration of Cho. We also found no significant change in the relationship between these neurotransmitters 
268 when we controlled for Cho (Fig. 2k; visual: $z 57=1.09, p=.274$; motor: $z_{47}=1.24$, $269 p=.215)$; however, controlling for Cho, like controlling for tNAA resulted in a significant 270 negative correlation between GABA + and Glx in visual ( $r_{58}=-.29, p=.026$ ) and motor 271 cortices $\left(r_{47}=-.30, p=.040\right)$. By contrast, although we found no difference in the 272 relationship between GABA+ and GIx in visual or motor cortices as a result of 273 controlling for the concentration of water (Fig. 21; visual: $z 57=0.08, p=.934$; motor: $274 z_{47}=0.57, p=.572$ ), controlling for water did not result in a significant negative 275 correlation in either area.

276 Post-hoc Bayesian analysis. In contrast to previous work examining medial parietal 277 cortex (Steel et al., 2020), we found no evidence for a positive correlation between 278 GABA+ and Glx in visual and motor cortices. In a post-hoc analysis, we assessed the 279 strength of the evidence for the null hypothesis $\left(\mathrm{H}_{0}\right)$, where the alternative hypothesis $280\left(\mathrm{H}_{1}\right)$ is that there is a positive relationship between GABA+ and Glx. The results of the 281 analysis are shown in Table 2. For the uncorrected GABA+ and Glx values, we find 282 moderate evidence in favour of $\mathrm{H}_{0}$ for both visual and motor cortices (Jeffreys, 1961). 283 For all corrected values, we find either moderate or strong evidence in favour of $\mathrm{H}_{0}$. 
Table 2. Probability of positive correlation between GABA+ and Glx

\begin{tabular}{|c|c|c|}
\hline \multirow[b]{2}{*}{ Confounding factor } & \multicolumn{2}{|c|}{ Bayes factor $\mathrm{BF}_{10}$} \\
\hline & visual & motor \\
\hline none & $3^{*}$ & $0.146^{*}$ \\
\hline age and & $0.103^{*}$ & $0.169^{*}$ \\
\hline frequency drift & $0.110^{*}$ & $0.162^{*}$ \\
\hline wate & * & $0.151^{*}$ \\
\hline tNA & 08 & $7^{*}$ \\
\hline GABA fit error & $0.101^{*}$ & $0.157^{\star}$ \\
\hline Glx fit error & $0.096^{* *}$ & $0.157^{*}$ \\
\hline grey $n$ & $0.101^{*}$ & $0.157^{\star}$ \\
\hline $\mathrm{tN}$ & 0.05 & $0.060^{* *}$ \\
\hline $\mathrm{tCr}$ concentration & $0.064^{* *}$ & $0.062^{\star *}$ \\
\hline Cho concentration & $0.053^{* *}$ & $0.062^{* *}$ \\
\hline water concentra & $0.096^{* *}$ & $0.092^{\star *}$ \\
\hline
\end{tabular}

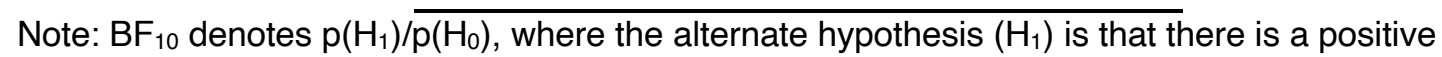
correlation between GABA+ and Glx; single-, and double-asterisks indicate moderate and strong evidence in favour of $\mathrm{H}_{0}$ (Jeffreys, 1961), respectively.

288 Visual stimulation control analysis. We found evidence that there is no positive correlation between GABA+ and Glx in visual and motor cortices. Consistent with previous work (Steel et al., 2020), spectra were acquired while participants were at rest. However, it is possible that a positive correlation between GABA+ and Glx in visual and motor cortices can only be observed during periods of relatively high activity. To test this possibility, we assessed the relationship between GABA+ and Glx 294 in visual cortex while participants received visual stimulation (see the 'mixed polarity' condition of (Rideaux et al., 2019) for a detailed description of the visual stimulus). Consistent with the results from participants at rest, we found no evidence of a 297 relationship between GABA+ and Glx in visual cortex of participants during visual stimulation $\left(r_{31}=.23, p=.213\right.$; Fig. 3). 
bioRxiv preprint doi: https://doi.org/10.1101/2021.03.02.433628; this version posted March 2, 2021. The copyright holder for this preprint (which was not certified by peer review) is the author/funder, who has granted bioRxiv a license to display the preprint in perpetuity. It is made available under aCC-BY 4.0 International license.

NO BALANCE BETWEEN GLX AND GABA IN VISUAL AND MOTOR CORTICES 16

299

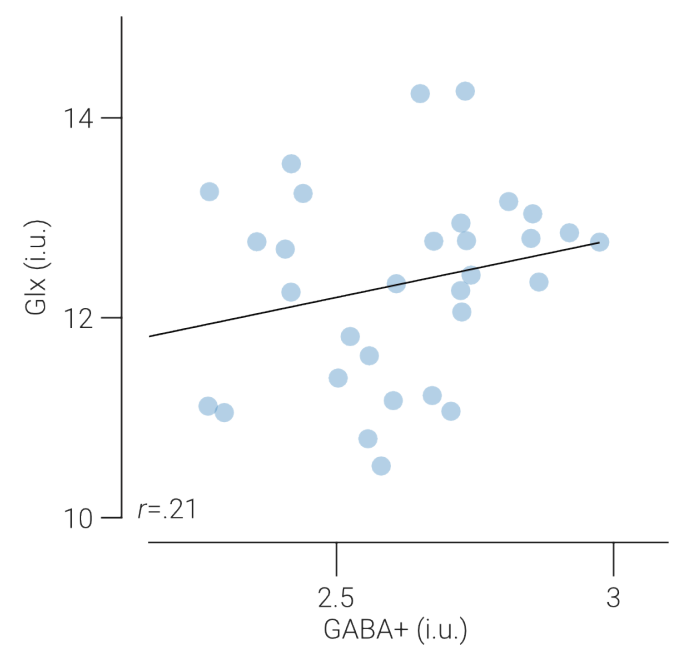

Figure 3. No balance between GABA+ and GIx in stimulated visual cortex. Glx concentration as a function of GABA+ concentration in visual cortices of participants while receiving visual stimulation.

GIx and GABA+ values are referenced to water and expressed in institutional units (i.u.). Line indicates best linear fit. 


\section{DISCUSSION}

301

302

303

304

305

306

307

308

309

310

311

312

313

314

315

316

317

318

319

320

321

322

323

324

325

326

327

328

329

330

331

Previous MRS work revealed a positive correlation between GABA+ and GIx in medial parietal cortex of resting human participants (Steel et al., 2020). Here we performed the same test in visual and motor cortices and found no correlation between these neurotransmitters in either region. Post-hoc Bayesian analyses yielded moderate to strong evidence in favour of the null hypothesis, that is, there is no positive correlation between GABA+ and Glx. These results show that the relationship between these neurotransmitters is regionally localized, rather than a brain-wide characteristic. Consistent with (Steel et al., 2020), we controlled for age, gender, signal quality and tissue composition, and found no change in the relationship between GABA+ and GIx. However, controlling for the concentration of other neurochemicals ( $t C r, t N A A, C h o$ ) revealed a negative correlation between these neurotransmitters in visual and motor cortices, suggesting a population-level imbalance in these regions.

These results appear to conflict with theoretical (Marín, 2012; Shadlen \& Newsome, 1994; Van Vreeswijk \& Sompolinsky, 1996; Vogels \& Abbott, 2009) and empirical (Haider, Duque, Hasenstaub, \& McCormick, 2006; Shu, Hasenstaub, \& McCormick, 2003) evidence supporting canonical E/I balance within the brain. This discrepancy underscores differences in how $\mathrm{E} / \mathrm{l}$ balance is measured. For example, in response to visual stimulation, electrophysiological work has shown concomitant excitatory and inhibitory activity (Anderson et al., 2000; Maffei \& Turrigiano, 2008; Monier, Chavane, Baudot, Graham, \& Frégnac, 2003; Priebe \& Ferster, 2005; Xue et al., 2014), indicating balance, whereas MRS studies show GABA and Glu/GIx change in opposite directions (Kurcyus et al., 2018; Mekle et al., 2017; Rideaux, 2020; Rideaux et al., 2019), indicating imbalance. The negative correlation between GABA+ and GIx, and their changing in opposite directions, may be related to the GABAglutamate cycle. In particular, at GABAergic synapses, Gln must be converted to Glu before being metabolised into GABA. Thus, increased GABA production could result in a temporary reduction in Gln and/or Glu, both of which comprise the Glx signal. These results do not undermine previous electrophysiological evidence for $E / I$ balance. Rather, they demonstrate a dissociation between electrophysiologically measured I/E balance and the ratio of MRS visible GABA+ and GIx (the source(s) of which remain unclear). 
Previous MRS work yielded inconsistent evidence for a balance between

GABA+ and Glx in early visual and motor cortices. Some studies have reported a significant positive correlation between GABA and Glx/Glu in visual (Rafique \& Steeves, 2020) or motor cortices (Rafique \& Steeves, 2020; Terhune, Russo, Near, Stagg, \& Cohen Kadosh, 2014), while others have failed to detect a relationship (Kurcyus et al., 2018; Terhune et al., 2014; Van Loon et al., 2013). These inconsistences may be a product of correlations based on relatively small sample sizes (Rafique \& Steeves, 2020; Terhune et al., 2014) and less consideration of confounding factors (e.g., voxel tissue composition). Here we employed a larger sample size and directly tested whether or not there is a balance between GABA+ and GIx in visual and motor cortices by assessing the influence of confounding factors and applying Bayesian statistics that can provide evidence for the null hypothesis.

344 Comparison of E/l observations in visual and motor cortices. The large body of evidence supporting $\mathrm{E} / \mathrm{l}$ balance is primarily based on electrophysiological work measuring changes in activity occurring over time at relatively high spatial (e.g., singlecell recording) and temporal resolution (e.g., milliseconds) (Haider et al., 2006; Okun \& Lampl, 2008; Shu et al., 2003); see (Isaacson \& Scanziani, 2011) for a review. By contrast, we measured the average concentration of excitatory (Glx) and inhibitory $(\mathrm{GABA}+)$ neurotransmitters from a relatively large cortical region (e.g., $18 \mathrm{~cm}^{3}$ ) over a $13 \mathrm{~min}$ period and assessed their relationship across individuals. The dissociation between the previously established E/l balance in these areas and the concentration of MRS visible excitatory and inhibitory neurotransmitters may be explained by differences in spatial/temporal resolution or observations made within and between individuals. For instance, while there may be no population-level common ratio between GABA and Glu, there may be intraindividual balance that can be observed by measuring the concentration of these neurotransmitters over time. We recently assessed the relationship between change in GABA+ and Glx in visual cortex at low and high temporal resolution using a novel analysis that increased the temporal resolution of neurochemical estimates detected with MRS by a factor of five (Rideaux, 2020). We found a predictive relationship between these neurotransmitters that could only be observed at high temporal resolution. That is, we found that a change in 
not suggest E/l balance, which would be evidenced by change in the same direction, the finding demonstrates that intraindividual relationships between these neurotransmitters can be detected with MRS, and further, that they can be obscured by the temporal resolution of the measurements.

Differences between brain regions. The discrepancy between our findings and those of (Steel et al., 2020) may be explained by differences in information processing between medial parietal cortex and visual and motor cortices. The function of visual and motor cortices is relatively specialised. The region of visual cortex targeted by our voxel contains primary and intermediate visual cortex (i.e., areas V1, V2 and V3). These areas primarily support processing of visual input from the retina via the lateral geniculate nucleus (Hubel \& Wiesel, 1959, 1968). Similarly, the cortical area targeted by our motor cortex voxel hosts the representation of hand and fingers and supports voluntary hand movement (Hluštík, Solodkin, Gullapalli, Noll, \& Small, 2001; Penfield \& Boldrey, 1937). Activity in sensorimotor areas may fluctuate more over time, due to natural variability in sensory inputs and motor activities (e.g., resting compared to running). In response to this variability, the $E / I$ balance in these areas may be maintained at a higher spatial and/or temporal scale than that measured in the current study. For instance, there is an abundance of evidence for this balance when measured at higher temporal and spatial resolution, as measured with electrophysiological techniques (Anderson et al., 2000; Dehghani et al., 2016; Maffei \& Turrigiano, 2008; Priebe \& Ferster, 2005; Wilent \& Contreras, 2005; Xue, Atallah, \& Scanziani, 2014). By contrast, the medial parietal lobe acts as an information processing hub; it is associated with a diverse range of perceptual and cognitive functions, including (but not limited to) visual scene perception (Epstein, Parker, \& Feiler, 2007; Silson, Steel, Kidder, Gilmore, \& Baker, 2019), memory recall (Wagner, Shannon, Kahn, \& Buckner, 2005), future prediction (Szpunar, Watson, \& McDermott, 2007), and heading direction (Baumann \& Mattingley, 2010). As such, its activity may be less variable and it may benefit from $E / l$ balance maintained at a temporal and/or spatial scale that is detectable with MRS measurements averaged over a $\sim 10$ min period.

Another, related, possible explanation is how these brain regions behave during rest. The medial parietal cortex is a core component of the default mode network 
396 (Andrews-Hanna, Reidler, Sepulcre, Poulin, \& Buckner, 2010), and is active during 397 rest and spontaneous thought (Fox, Spreng, Ellamil, Andrews-Hanna, \& Christoff, 398 2015; Raichle, 2015). By contrast, visual and motor cortices are active during visual 399 stimulation and motor movement, respectively, and relatively inactive while at rest. 400 Thus, another possible explanation for the discrepancy in the relationship between 401 GABA+ and Glx is that balance can only be observed during periods of relatively high 402 neural activity. However, this explanation seems unlikely, as we found no evidence for 403 a relationship between these neurotransmitters in visual cortex of participants who 404 received visual stimulation. Further, electrophysiological work suggests excitatory and 405 inhibitory activity in sensory cortices is balanced even during spontaneous activity 406 (Atallah \& Scanziani, 2009; Okun \& Lampl, 2008).

407 No clear link to metabolism. MRS-detected GABA and Glx/Glutamate is typically 408 interpreted as indicator of inhibitory and excitatory neural activity (Edden, 409 Muthukumaraswamy, Freeman, \& Singh, 2009; Lunghi, Emir, Morrone, \& Bridge, 410 2015; Stagg, Bachtiar, \& Johansen-Berg, 2011a); however, the source(s) that give rise 411 to the neurochemical signal that is detected remain unclear (Stagg, Bachtiar, \& 412 Johansen-Berg, 2011b). Steel et al. (2020) found that correlation between GABA+ and 413 Glx was reduced when the concentration of $\mathrm{tCr}$ and $\mathrm{tNAA}$, but not Cho, were 414 controlled. The authors interpreted this as indicative of being related to metabolic 415 function, as NAA is found exclusively within neurons (Urenjak, Williams, Gadian, \& 416 Noble, 1992) and $\mathrm{Cr}$ is involved in metabolic processes within multiple cell types 417 (Andres et al., 2008); however, note that Cho is the precursor for the neurotransmitter 418 acetylcholine (Nachmansohn \& Machado, 1943). By contrast, we found that controlling 419 for all three of these neurochemicals (tCr, tNAA, and Cho) had a similar effect on the 420 relationship between GABA+ and GIx, producing more negative correlations (although 421 controlling for $\mathrm{tCr}$ did not produce a significant correlation in visual cortex). Thus, our 422 results suggest a different explanation, that is, that GABA+ and Glx share variance 423 with all the neurochemical concentration measurements from the same spectrum. 424 Here, and in Steel et al. (2020), the concentration of water was measured from 425 separate (water unsuppressed) spectra and thus may have independent variance. 426 Thus, while the results of Steel et al. (2020) appear are consistent with a link to 
metabolism, our results are more parsimoniously explained by factors that influence the entire spectra (e.g., participant movement).

429 Clinical relevance. An E/l imbalance, in particular between Glu and GABA, is 430 associated with a range of psychiatric and neurological illnesses including autism 431 (Chao et al., 2010; Markram \& Markram, 2010; Robertson, Ratai, \& Kanwisher, 2016; 432 Rubenstein \& Merzenich, 2003; Vattikuti \& Chow, 2010), schizophrenia (Kehrer et al., 433 2008), and epilepsy (Bradford, 1995; Olsen \& Avoli, 1997). Evidence from MRS 434 studies has revealed associations between GIx, GABA, and the severity of symptoms 435 associated with autism spectrum disorder and schizophrenia (Brown et al., 2013; Egerton et al., 2012; Horder et al., 2013; Smesny et al., 2015), see (Foss-Feig et al., 2017) for a review. However, these associations appear to be regionally variable. For instance, autism spectrum disorder is associated with increased Glx in auditory cortex (Brown et al., 2013) and decreased Glx in basal ganglia (Horder et al., 2013). The discrepancy between the results from medial parietal cortex (Steel et al., 2020) and our findings from visual and motor cortices seem to be consistent with the regional variability reported in previous clinical MRS studies and highlight the diversity of relationships between neurochemicals, and neurochemicals and behaviour, across the brain (Greenhouse, Noah, Maddock, \& Ivry, 2016).

Limitations. Glutamate is the primary excitatory neurotransmitter in the central nervous system, whereas here we measured Glx, a complex comprising both glutamate and glutamine (Puts \& Edden, 2012; Ramadan, Lin, \& Stanwell, 2013). MRS work using phantoms indicates that glutamate contributes at least half the Glx signal in MEGA-PRESS difference spectra (Shungu et al., 2013; van Veenendaal et al., 2018), so it seems reasonable to interpret the concentration of Glx as representative of glutamate. However, it is possible that the contribution of glutamine to the Glx signal masked a correlation between GABA and glutamate. That is, a negative correlation between glutamine and GABA+ could have obscured a positive correlation between 454 glutamate and GABA+, when combined in the Glx signal. Future work is needed test this possibility by directly measuring glutamate.

456 Conclusion. We measured the concentration of GABA+ and GIx in visual and motor cortices and found moderate to strong evidence that there is no positive correlation 
458 between these neurotransmitters. These results contrast previous work that found a 459 positive relationship between these neurotransmitters in medial parietal cortex and 460 shows that there is no brain-wide interindividual balance between the concentration of 461 GABA+ and Glx. Given that a balance was only found in one of three cortical locations, 462 this suggests it may be the exception, rather than the rule. Our findings indicate a 463 dissociation between the well-established E/l balance supported by previous 464 theoretical (Marín, 2012; Shadlen \& Newsome, 1994; Van Vreeswijk \& Sompolinsky, 465 1996; Vogels \& Abbott, 2009) and empirical evidence (Haider et al., 2006; Okun \& 466 Lampl, 2008; Shu et al., 2003) and the ratio between MRS-detected excitatory and 467 inhibitory transmitters. This dissociation has implications for studies using this ratio as 468 a proxy for E/l balance (Bang et al., 2018; Gu et al., 2019; Koizumi et al., 2018; Shibata 469 et al., 2017; Takei et al., 2016), and for understanding and diagnosis of neurological 470 and psychiatric illnesses characterised by an imbalance in excitation and inhibition. 


\section{REFERENCES}

472 Anderson, J. S., Carandini, M., \& Ferster, D. (2000). Orientation tuning of input conductance, excitation, and inhibition in cat primary visual cortex. Journal of Neurophysiology, 84(2), 909-926. https://doi.org/10.1152/jn.2000.84.2.909

Andres, R. H., Ang', A., Ducray, A. D., Schlattner, U., Wallimann, T., \& Widmer, H. R. (2008). Functions and effects of creatine in the central nervous system. Brain Research Bulletin, 76, 329-343.

Andrews-Hanna, J. R., Reidler, J. S., Sepulcre, J., Poulin, R., \& Buckner, R. L. (2010). Functional-Anatomic Fractionation of the Brain's Default Network.

Ashburner, J., \& Friston, K. J. (2005). Unified segmentation. Neurolmage, 26(3), 839-851. https://doi.org/10.1016/j.neuroimage.2005.02.018

Atallah, B. V., \& Scanziani, M. (2009). Instantaneous Modulation of Gamma Oscillation Frequency by Balancing Excitation with Inhibition. Neuron, 62(4), 566-577. https://doi.org/10.1016/j.neuron.2009.04.027

Bang, J. W., Shibata, K., Frank, S. M., Walsh, E. G., Greenlee, M. W., Watanabe, T., neurochemical mechanisms. Nature Human Behaviour, 2(7), 507-513. https://doi.org/10.1038/s41562-018-0366-8

Baumann, O., \& Mattingley, J. B. (2010). Medial parietal cortex encodes perceived heading direction in humans. Journal of Neuroscience, 30(39), 12897-12901. https://doi.org/10.1523/JNEUROSCI.3077-10.2010

Bezalel, V., Paz, R., \& Tal, A. (2019). Inhibitory and excitatory mechanisms in the human cingulate-cortex support reinforcement learning: A functional Proton Magnetic Resonance Spectroscopy study. Neurolmage, 184, 25-35. https://doi.org/10.1016/j.neuroimage.2018.09.016

Bradford, H. F. (1995). Glutamate, GABA and epilepsy. Progress in Neurobiology, 47(6), 477-511. https://doi.org/10.1016/0301-0082(95)00030-5 concentration in the auditory cortex of persons with autism and first-degree relatives: A 1H-MRS study. Autism Research, 6(1), 1-10. 
Cassady, K., Gagnon, H., Lalwani, P., Simmonite, M., Foerster, B., Park, D., ... Polk, to GABA and to sensorimotor performance. Neurolmage, 186, 234-244.

507 https://doi.org/10.1016/j.neuroimage.2018.11.008

508

Chao, H. T., Chen, H., Samaco, R. C., Xue, M., Chahrour, M., Yoo, J., ... Zoghbi, H. Y. (2010). Dysfunction in GABA signalling mediates autism-like stereotypies and Rett syndrome phenotypes. Nature, 468(7321), 263-269. https://doi.org/10.1038/nature09582

Dehghani, N., Peyrache, A., Telenczuk, B., Le Van Quyen, M., Halgren, E., Cash, S. S., ... Destexhe, A. (2016). Dynamic Balance of Excitation and Inhibition in Human and Monkey Neocortex. Nature Publishing Group. https://doi.org/10.1038/srep23176 (2009). Orientation Discrimination Performance Is Predicted by GABA Concentration and Gamma Oscillation Frequency in Human Primary Visual Cortex. Journal of Neuroscience, 29(50), 15721-15726. https://doi.org/10.1523/JNEUROSCI.4426-09.2009

Edden, R. A. E., Puts, N. A. J., Harris, A. D., Barker, P. B., \& Evans, C. J. (2014). for parahippocampal and retrosplenial cortices in place recognition. Journal of Neuroscience, 27(23), 6141-6149. https://doi.org/10.1523/JNEUROSCI.0799-

534 Erdfelder, E., Faul, F., \& Buchner, A. (1996). GPOWER: A general power analysis 
program. Behavior Research Methods, Instruments, and Computers, 28(1), 1-

Foss-Feig, J. H., Adkinson, B. D., Ji, J. L., Yang, G., Srihari, V. H., McPartland, J. C., ... Anticevic, A. (2017). Searching for Cross-Diagnostic Convergence: Neural Mechanisms Governing Excitation and Inhibition Balance in Schizophrenia and Autism Spectrum Disorders. Biological Psychiatry, 81(10), 848-861. https://doi.org/10.1016/j.biopsych.2017.03.005 of mind-wandering and related spontaneous thought processes. Neurolmage, 111, 611-621. https://doi.org/10.1016/j.neuroimage.2015.02.039

Ganji, S. K., Banerjee, A., Patel, A. M., Zhao, Y. D., Dimitrov, I. E., Browning, J. D., ... Choi, C. (2012). T 2 measurement of J-coupled metabolites in the human brain at 3T. NMR in Biomedicine, 25(4), 523-529. https://doi.org/10.1002/nbm.1767

Gao, F., Edden, R. A. E., Li, M., Puts, N. A. J., Wang, G., Liu, C., ... Barker, P. B. (2013). Edited magnetic resonance spectroscopy detects an age-related decline in brain GABA levels. Neurolmage, 78, 75-82. https://doi.org/10.1016/j.neuroimage.2013.04.012

Gasparovic, C., Song, T., Devier, D., Bockholt, H. J., Caprihan, A., Mullins, P. G., ... proton spectroscopic imaging. Magnetic Resonance in Medicine, 55(6), 12191226. https://doi.org/10.1002/mrm.20901

Greenhouse, I., Noah, S., Maddock, R. J., \& Ivry, R. B. (2016). Individual differences in GABA content are reliable but are not uniform across the human cortex. Neurolmage, 139, 1-7. https://doi.org/10.1016/j.neuroimage.2016.06.007 balance predicts default-mode network deactivation via functional connectivity. Neurolmage, 185, 388-397. https://doi.org/10.1016/j.neuroimage.2018.10.055 inhibition. Journal of Neuroscience, 26(17), 4535-4545. 
Harris, A. D., Glaubitz, B., Near, J., John Evans, C., Puts, N. A. J., Schmidt-Wilcke, T., ... Edden, R. A. E. (2014). Impact of frequency drift on gamma-aminobutyric acid-edited MR spectroscopy. Magnetic Resonance in Medicine, 72(4), 941948. https://doi.org/10.1002/mrm.25009

Harris, A. D., Puts, N. A. J., \& Edden, R. A. E. (2015). Tissue correction for GABAedited MRS: Considerations of voxel composition, tissue segmentation, and tissue relaxations. Journal of Magnetic Resonance Imaging, 42(5), 1431-1440. https://doi.org/10.1002/jmri.24903

Higley, M. J., \& Contreras, D. (2006). Balanced excitation and inhibition determine spike timing during frequency adaptation. Journal of Neuroscience, 26(2), 448457. https://doi.org/10.1523/JNEUROSCI.3506-05.2006

Hluštík, P., Solodkin, A., Gullapalli, R. P., Noll, D. C., \& Small, S. L. (2001).

580 Somatotopy in human primary motor and somatosensory hand representations revisited. Cerebral Cortex, 11(4), 312-321. https://doi.org/10.1093/cercor/11.4.312

Horder, J., Lavender, T., Mendez, M. A., O’Gorman, R., Daly, E., Craig, M. C., ... Murphy, D. G. (2013). Reduced subcortical glutamate/glutamine in adults with autism spectrum disorders: A [1H]MRS study. Translational Psychiatry, 3(April), 7-9. https://doi.org/10.1038/tp.2013.53

Hubel, D. H., \& Wiesel, T. N. (1959). Receptive fields of single neurones in the cat's 589 striate cortex. The Journal of Physiology, 148(3), 574-591. https://doi.org/10.1113/jphysiol.1959.sp006308

Hubel, D. H., \& Wiesel, T. N. (1968). Receptive fields and functional architecture of monkey striate cortex. The Journal of Physiology, 195(1), 215-243. https://doi.org/10.1113/jphysiol.1968.sp008455 excitation and inhibition in the human visual cortex - a combined fMRI-MR Spectroscopy study at 7 Tesla. In Proceedings of the International Society for Magnetic Resonance in Medicine. 
599

600

601

602

603

604

605

606

607

608

609

610

611

612

613

614

615

616

617

618

619

620

621

622

623

624

625

626

627

628

629

630

Jeffreys, H. (1961). Theory of probability (3rd ed.). Oxford University Press, New York, NY. Retrieved from

https://catalogue.nla.gov.au/Record/759870/Export?style=bibtex

Kapogiannis, D., Reiter, D. A., Willette, A. A., \& Mattson, M. P. (2013).

Posteromedial cortex glutamate and GABA predict intrinsic functional

connectivity of the default mode network. Neurolmage, 64(1), 112-119.

https://doi.org/10.1016/j.neuroimage.2012.09.029

Kehrer, C., Maziashvili, N., Dugladze, T., \& Gloveli, T. (2008). Altered excitatoryinhibitory balance in the NMDA-hypofunction model of schizophrenia. Frontiers in Molecular Neuroscience, 1(APR). https://doi.org/10.3389/neuro.02/006.2008

Koizumi, A., Lau, H., Shimada, Y., \& Kondo, H. M. (2018). The effects of neurochemical balance in the anterior cingulate cortex and dorsolateral prefrontal cortex on volitional control under irrelevant distraction. Consciousness and Cognition, 59(February), 104-111.

https://doi.org/10.1016/j.concog.2018.01.001

Kurcyus, K., Annac, E., Hanning, N. M., Harris, A. D., Oeltzschner, G., Edden, R. A. E., \& Riedl, V. (2018). Opposite Dynamics of GABA and Glutamate Levels in the Occipital Cortex during Visual Processing. The Journal of Neuroscience, 38(46), 9967-9976. https://doi.org/10.1523/JNEUROSCI.1214-18.2018

Lunghi, C., Emir, U. E., Morrone, M. C., \& Bridge, H. (2015). Short-Term monocular deprivation alters GABA in the adult human visual cortex. Current Biology. https://doi.org/10.1016/j.cub.2015.04.021

Maffei, A., \& Turrigiano, G. G. (2008). Multiple modes of network homeostasis in visual cortical layer 2/3. Journal of Neuroscience, 28(17), 4377-4384. https://doi.org/10.1523/JNEUROSCI.5298-07.2008

Marín, O. (2012). Interneuron dysfunction. https://doi.org/10.1038/nrn3155

Markram, K., \& Markram, H. (2010). The intense world theory - A unifying theory of the neurobiology of autism. Frontiers in Human Neuroscience, 4(December), 129. https://doi.org/10.3389/fnhum.2010.00224

Mekle, R., Kühn, S., Pfeiffer, H., Aydin, S., Schubert, F., \& Ittermann, B. (2017). Detection of metabolite changes in response to a varying visual stimulation paradigm using short-TE $1 \mathrm{H}$ MRS at 7 T. NMR in Biomedicine, 30(2), e3672. 
Mescher, M., Merkle, H., Kirsch, J., Garwood, M., \& Gruetter, R. (1998).

Mikkelsen, M., Rimbault, D. L., Barker, P. B., Bhattacharyya, P. K., Brix, M. K., Buur, P. F., ... Edden, R. A. E. (2019). Big GABA II: Water-referenced edited MR spectroscopy at 25 research sites. Neurolmage, 191, 537-548. https://doi.org/10.1016/J.NEUROIMAGE.2019.02.059

Monier, C., Chavane, F., Baudot, P., Graham, L. J., \& Frégnac, Y. (2003). Orientation and direction selectivity of synaptic inputs in visual cortical neurons: A diversity of combinations produces spike tuning. Neuron, 37(4), 663-680. https://doi.org/10.1016/S0896-6273(03)00064-3

Nachmansohn, D., \& Machado, A. L. (1943). The formation of acetylcholine. A new enzyme: "choline acetylase." Journal of Neurophysiology, 6(5), 397-403.

O'Gorman, R. L., Michels, L., Edden, R. A. E., Murdoch, J. B., \& Martin, E. (2011). In vivo detection of GABA and glutamate with MEGA-PRESS: Reproducibility and gender effects. Journal of Magnetic Resonance Imaging, 33(5), 1262-1267. https://doi.org/10.1002/jmri.22520

Okun, M., \& Lampl, I. (2008). Instantaneous correlation of excitation and inhibition during ongoing and sensory-evoked activities. NATURE NEUROSCIENCE VOLUME, 11(5). https://doi.org/10.1038/nn.2105

Olsen, R. W., \& Avoli, M. (1997). GABA and Epileptogenesis. Epilepsia, 38(4), 399407. https://doi.org/10.1111/j.1528-1157.1997.tb01728.x

Penfield, W., \& Boldrey, E. (1937). Somatic Motor and Sensory Representation in Man. Brain, 389-443.

Petroff, O. A. C., Ogino, T., \& Alger, J. R. (1988). High-Resolution Proton Magnetic Resonance Spectroscopy of Rabbit Brain: Regional Metabolite Levels and Postmortem Changes. Journal of Neurochemistry, 51(1), 163-171. https://doi.org/10.1111/j.1471-4159.1988.tb04850.x 
Posse, S., Otazo, R., Caprihan, A., Bustillo, J., Chen, H., Henry, P.-G., ... Alger, J.

Priebe, N. J., \& Ferster, D. (2005). Direction selectivity of excitation and inhibition in simple cells of the cat primary visual cortex. Neuron, 45(1), 133-145. https://doi.org/10.1016/j.neuron.2004.12.024

Puts, N. A. J., \& Edden, R. A. E. (2012). In vivo magnetic resonance spectroscopy of GABA: A methodological review. Progress in Nuclear Magnetic Resonance Spectroscopy, 60, 29-41. https://doi.org/10.1016/j.pnmrs.2011.06.001

Rafique, S. A., \& Steeves, J. K. E. (2020). Assessing differential effects of single and accelerated low-frequency rTMS to the visual cortex on GABA and glutamate concentrations. Brain and Behavior, (May), 1-18. https://doi.org/10.1002/brb3.1845

Ramadan, S., Lin, A., \& Stanwell, P. (2013). Glutamate and glutamine: A review of in 682 vivo MRS in the human brain. NMR in Biomedicine, 26(12), 1630-1646. https://doi.org/10.1002/nbm.3045

Rideaux, R. (2020). Temporal dynamics of GABA and Glx in the visual cortex. ENeuro, ENEURO.0082-20.2020. https://doi.org/10.1523/eneuro.0082-20.2020 dot stereograms alter GABA and Glx concentration in the early visual cortex. Journal of Neurophysiology, 122(2), 888-896. integration by suppression in human visual cortex. Nature Communications, 9(1), 1502. https://doi.org/10.1038/s41467-018-03400-y in the Autistic Brain. Current Biology, 26(1), 80-85. 
Rubenstein, J. L. R., \& Merzenich, M. M. (2003). Model of autism: increased ratio of excitation/inhibition in key neural systems. Genes Brain \& Behavior, 2(5), 255267. https://doi.org/10.1046/j.1601-183X.2003.00037.x

Sailasuta, N., Ernst, T., \& Chang, L. (2008). Regional variations and the effects of

700

701

702 age and gender on glutamate concentrations in the human brain. Magnetic Resonance Imaging, 26(5), 667-675. https://doi.org/10.1016/j.mri.2007.06.007

Shadlen, M. N., \& Newsome, W. T. (1994). Noise, neural codes and cortical

Shibata, K., Sasaki, Y., Bang, J. W., Walsh, E. G., Machizawa, M. G., Tamaki, M., ... Watanabe, T. (2017). Overlearning hyperstabilizes a skill by rapidly making neurochemical processing inhibitory-dominant. Nature Neuroscience, 20(3), 470-475. https://doi.org/10.1038/nn.4490

Shungu, D. C., Mao, X., Gu, M., Milak, M. S., Weiduschat, N., Mayer, D., ... Kegeles, L. S. (2013). 'Glx' Measured by J-editing/MEGA-PRESS is Primarily 'Pure' Glutamate...Or is it? Proc. Intl. Soc. Mag. Reson. Med. 21, 21, 3985. Retrieved from http://cds.ismrm.org/protected/13MProceedings/files/3985.PDF

Silson, E. H., Steel, A., Kidder, A., Gilmore, A. W., \& Baker, C. I. (2019). Distinct subdivisions of human medial parietal cortex support recollection of people and places. ELife, 8. https://doi.org/10.7554/eLife.47391

Smesny, S., Gussew, A., Biesel, N. J., Schack, S., Walther, M., Rzanny, R., ... Reichenbach, J. R. (2015). Glutamatergic dysfunction linked to energy and membrane lipid metabolism in frontal and anterior cingulate cortices of never treated first-episode schizophrenia patients. Schizophrenia Research, 168(1-2), 322-329. https://doi.org/10.1016/j.schres.2015.07.013

Stagg, C. J., Bachtiar, V., \& Johansen-Berg, H. (2011a). The role of GABA in human motor learning. Current Biology, 21(6), 480-484. https://doi.org/10.1016/j.cub.2011.01.069 Stagg, C. J., Bachtiar, V., \& Johansen-Berg, H. (2011b). What are we measuring 

with GABA Magnetic Resonance Spectroscopy? Communicative \& Integrative Biology, 4(5), 573-575. https://doi.org/10.4161/cib.16213

729

730

731

732

733

734

735

736

737

738

739

740

741

742

743

744

745

746

747

748

749

750

751

752

753

754

755

756

757

758

Steel, A., Mikkelsen, M., Edden, R. A. E., \& Robertson, C. E. (2020). Regional balance between glutamate+glutamine and GABA+ in the resting human brain. Neurolmage, 220, 117112. https://doi.org/10.1016/j.neuroimage.2020.117112

Szpunar, K. K., Watson, J. M., \& McDermott, K. B. (2007). Neural substrates of envisioning the future. Proceedings of the National Academy of Sciences of the United States of America (Vol. 104). https://doi.org/10.1073/pnas.0610082104

Takei, Y., Fujihara, K., Tagawa, M., Hironaga, N., Near, J., Kasagi, M., ... Fukuda, M. (2016). The inhibition/excitation ratio related to task-induced oscillatory modulations during a working memory task: A multtimodal-imaging study using MEG and MRS. Neurolmage, 128, 302-315.

https://doi.org/10.1016/j.neuroimage.2015.12.057

Terhune, D. B., Russo, S., Near, J., Stagg, C. J., \& Cohen Kadosh, R. (2014). GABA Predicts Time Perception. Journal of Neuroscience, 34(12), 4364-4370. https://doi.org/10.1523/JNEUROSCI.3972-13.2014

Tkáč, I., \& Gruetter, R. (2005). Methodology of 1H NMR spectroscopy of the human brain at very high magnetic fields. Applied Magnetic Resonance, 29(1), 139157. https://doi.org/10.1007/BF03166960

Urenjak, J., Williams, S. R., Gadian, D. G., \& Noble, M. (1992). Specific Expression of N-Acetylaspartate in Neurons, Oligodendrocyte-Type-2 Astrocyte Progenitors, and Immature Oligodendrocytes In Vitro. Journal of Neurochemistry, 59(1), 5561. https://doi.org/10.1111/j.1471-4159.1992.tb08875.x

Van Loon, A. M., Knapen, T., Scholte, H. S., St. John-Saaltink, E., Donner, T. H., \& Lamme, V. A. F. (2013). GABA shapes the dynamics of bistable perception. Current Biology, 23(9), 823-827. https://doi.org/10.1016/j.cub.2013.03.067 van Veenendaal, T. M., Backes, W. H., van Bussel, F. C. G., Edden, R. A. E., Puts, N. A. J., Aldenkamp, A. P., \& Jansen, J. F. A. (2018). Glutamate quantification by PRESS or MEGA-PRESS: Validation, repeatability, and concordance. Magnetic Resonance Imaging, 48, 107-114. https://doi.org/10.1016/j.mri.2017.12.029

Van Vreeswijk, C., \& Sompolinsky, H. (1996). Chaos in neuronal networks with 

balanced excitatory and inhibitory activity. Science, 274(5293), 1724-1726. https://doi.org/10.1126/science.274.5293.1724

761

762

763

764

765

766

767

768

769

770

771

772

773

774

775

776

777

778

779

780

781

782

783

784

Vattikuti, S., \& Chow, C. C. (2010). A Computational Model for Cerebral Cortical Dysfunction in Autism Spectrum Disorders. Biological Psychiatry, 67(7), 672678. https://doi.org/10.1016/j.biopsych.2009.09.008

Vogels, T. P., \& Abbott, L. F. (2009). Gating multiple signals through detailed balance of excitation and inhibition in spiking networks. NATURE NEUROSCIENCE VOLUME, 12(4). https://doi.org/10.1038/nn.2276

Wagenmakers, E.-J., Love, J., Marsman, M., Tahira Jamil, ·, Ly, · Alexander, Verhagen, J., ... Morey, D. (2018). Bayesian inference for psychology. Part II: Example applications with JASP. Psychon Bull Rev, 25, 58-76. https://doi.org/10.3758/s13423-017-1323-7

Wagner, A. D., Shannon, B. J., Kahn, I., \& Buckner, R. L. (2005). Parietal lobe contributions to episodic memory retrieval. Trends in Cognitive Sciences, 9(9), 445-453. https://doi.org/10.1016/j.tics.2005.07.001

Wehr, M., \& Zador, A. M. (2003). Balanced inhibition underlies tuning and sharpens spike timing in auditory cortex. Nature (Vol. 426). Retrieved from http://www.college-de-france.fr/media/jean-marietarascon/UPL58650_hydrogenecollege.pdf\%0Ahttp://www.sandia.gov/ jytsao/S olar FAQs.pdf\%0Apapers3://publication/uuid/E7A575F7-4BEF-4981-8E02277673CABC12\%0Awww.cea.fr/presse\%0Ahttp://dx.doi.org/10.1016/S1573-

Wilent, W. B., \& Contreras, D. (2005). Dynamics of excitation and inhibition underlying stimulus selectivity in rat somatosensory cortex. Nature Neuroscience, 8(10), 1364-1370. https://doi.org/10.1038/nn1545 Xue, M., Atallah, B. V, \& Scanziani, M. (2014). Equalizing excitation-inhibition ratios across visual cortical neurons. Nature. https://doi.org/10.1038/nature13321 